\title{
Ruptured hemorrhagic bulla in a patient with a HeartMate 3 treated with an Amplatzer device
}

\section{Bulla hemorrágica rota en un paciente con un HeartMate 3 tratado con un dispositivo Amplatzer}

\author{
Adrian daSilva-deAbreu, ${ }^{1,2 *}$, Oscar Maitas ${ }^{1,2}$, Juan P. Rodriguez-Escudero ${ }^{1}$, Sapna Desai, ${ }^{1,2}$, \\ Clement Eiswirth ${ }^{1,2}$, Selim Krim ${ }^{1,2}$, Hamang Patel1,2, Hector O. Ventura ${ }^{1,2}$, and Stacy A. Mandras ${ }^{1,2}$ \\ ${ }^{1}$ Department of Cardiovascular Diseases, John Ochsner Heart and Vascular Institute, Ochsner Medical Center, New Orleans, LA, USA; ${ }^{2}$ Ochsner \\ Clinical School, Faculty of Medicine, The University of Queensland, New Orleans, LA, USA.
}

A 60-year-old female with underlying emphysema and left ventricular assist device (LVAD) HeartMate 3 presented with progressive hemoptysis, dyspnea, and right chest pain. Baseline hemoglobin was $11.1 \mathrm{~g} / \mathrm{dL}$ and INR 2.9. A chest computed tomography showed enlargement of a right lung bulla with internal hemorrhage, measuring $105.6 \mathrm{~mm}$ in its widest diameter, and fluid-air level in its more cephalic portion (Fig. 1). Anticoagulation was held and cardiothoracic surgery consulted. The $2^{\text {nd }}$ day, the LVAD had low-flow alarms, her hemoglobin decreased to $6.1 \mathrm{~g} / \mathrm{dL}$ and she developed hypovolemic shock and hypoxic respiratory failure requiring intubation, blood transfusion, and inotrope/vasopressor therapy. A chest tube was placed for hemothorax, draining 2.7 $\mathrm{L}$ of blood. Angiography showed no active contrast extravasation but the hemothorax persisted through the next day. A second angiography was performed, during which a Montefiore pigtail catheter was advanced over a 0.035 in wire into the right pulmonary artery and diagnostic angiography was performed. Although no extravasation was evidenced, it was empirically decided to plug the right lower lobe artery to halt further hemothorax, for which a $18 \mathrm{~mm}$ Amplatzer Vascular Plug II was deemed to provide appropriate occlusion. The catheter and sheath were was exchanged for a 7 French by $100 \mathrm{~cm}$ Ansel sheath, which was advanced into the right lower lobe pulmonary artery. An $18 \mathrm{~mm}$ Amplatzer Vascular Plug II was advanced through the sheath and successfully deployed in the right lower lobe pulmonary artery (Fig. 2), and the hemorrhagic output decreased. She underwent surgical washout with removal of $1 \mathrm{~L}$ of clot. Her admission also complicated with an acute ischemic stroke and ventilator-associated pneumonia and was discharged on hospital day 32 without residual neurological deficits.

Bleeding and thrombosis are seen less frequently with centrifugal-flow pumps such as HeartMate $3^{1}$; nonetheless, bleeding complications continue to pose significant morbidity and mortality for patients with LVADs. This case highlights the importance of coordinated multidisciplinary management and the ingenious use of transcatheter interventions, such as pulmonary arterial occlusion with Amplatzer devices, to achieve bleeding-source control in patients with LVADs.

\section{Correspondence:}

*Adrian daSilva-deAbreu

E-mail: adrianjdasilva@gmail.com
Date of reception: 03-07-2020

Date of acceptance: 20-11-2020 DOI: 10.24875/ACME.M21000240
Available online: 23-03-2021 Arch Cardiol Mex (Eng). 2021;91(3):337-338 www.archivoscardiologia.com 2604-7063 / @ 2020 Instituto Nacional de Cardiología Ignacio Chávez. Published by Permanyer. This is an open access article under the CC BY-NC-ND license (http://creativecommons.org/licenses/by-nc-nd/4.0/). 


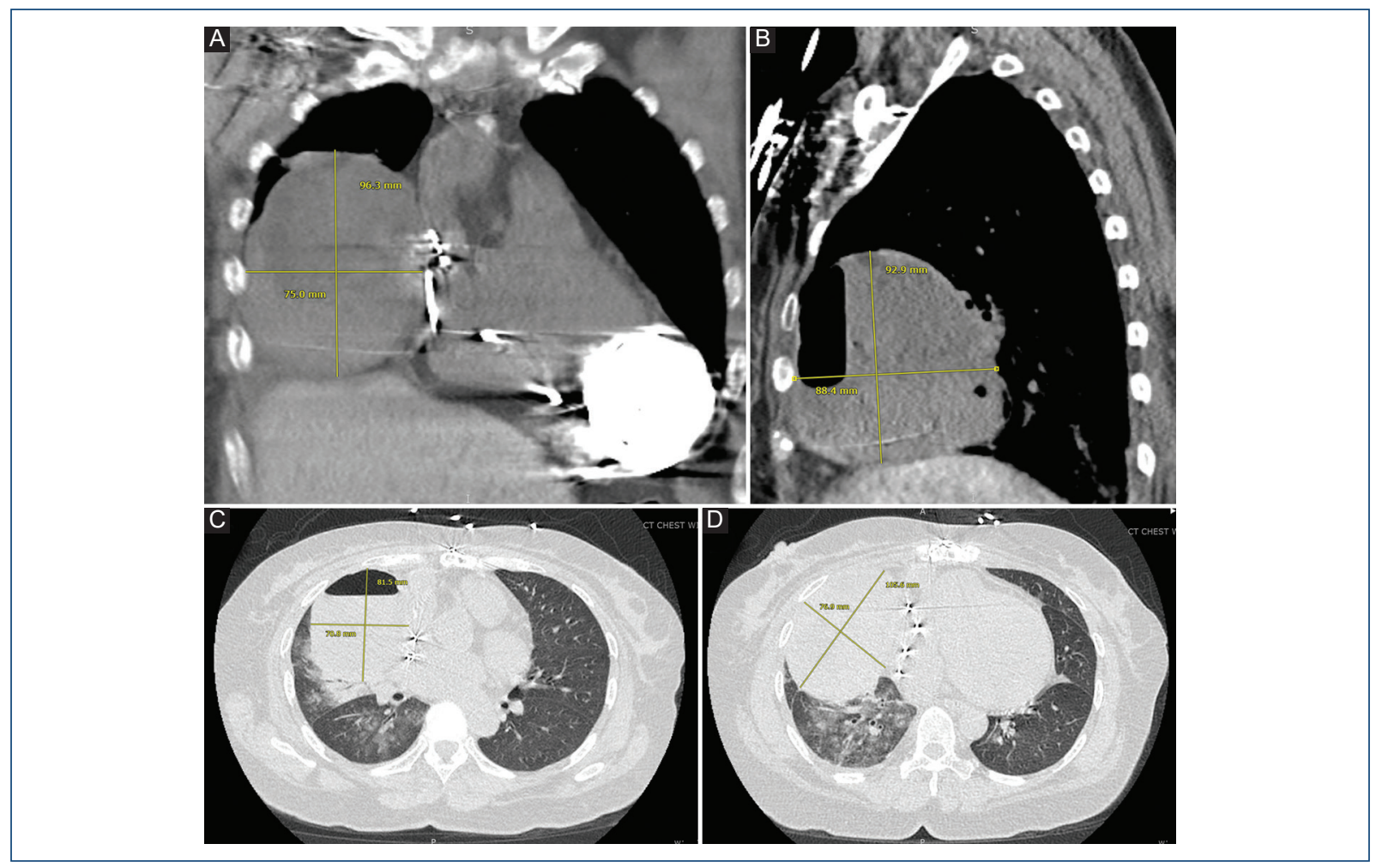

Figure 1. Chest computed tomography evidencing a large right lung bulla with internal hemorrhage (panels $A-D)$. The bulla measured $105.6 \mathrm{~mm}$ in its widest diameter, and there was a fluid-air level in its more cephalic portion.

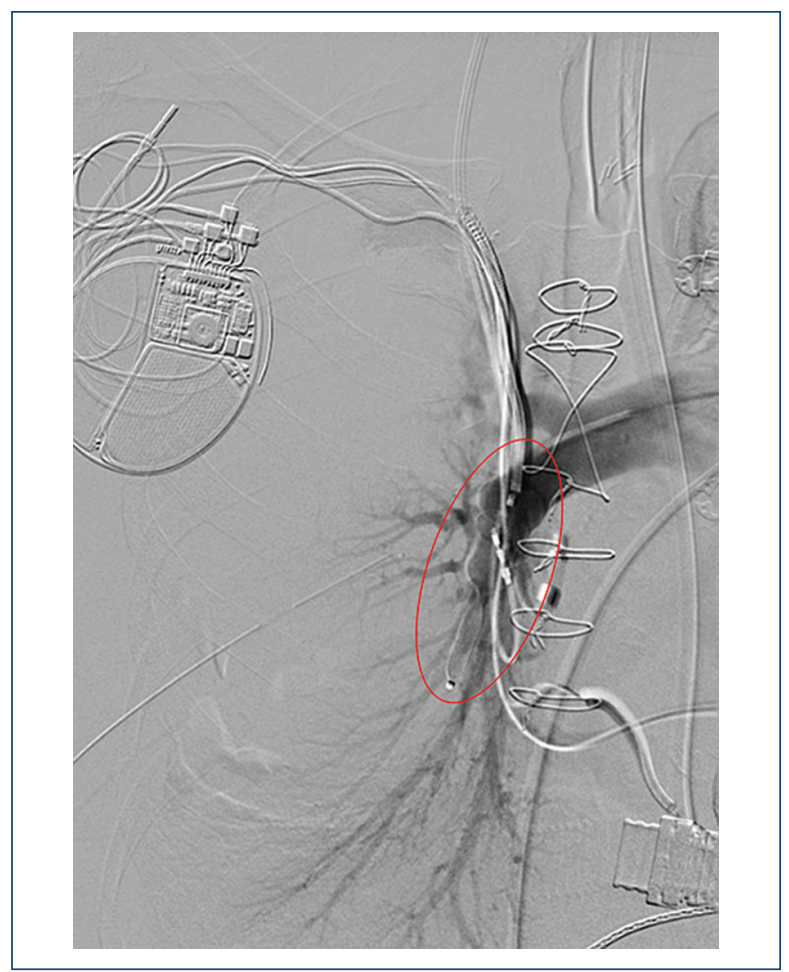

Figure 2. Post-procedural fluoroscopy. An 18-mm Amplatzer device was deployed in the right inferior pulmonary artery successfully decreasing distal flow and halting extravasation of blood.

\section{Funding}

None.

\section{Conflicts of interest}

The declare no conflicts of interests.

\section{Ethical disclosures}

Protection of human and animal subjects. The authors declare that no experiments were performed on humans or animals for this study.

Confidentiality of data. The authors declare that they have followed the protocols of their work center on the publication of patient data.

Right to privacy and informed consent. The authors declare that no patient data appear in this article.

\section{References}

1. Mehra MR, Uriel N, Naka Y, Cleveland JC, Yuzefpolskaya M, Salerno CT, et al. A fully magnetically levitated left ventricular assist device-final report. N Engl J Med. 2019;380:1618-27. 